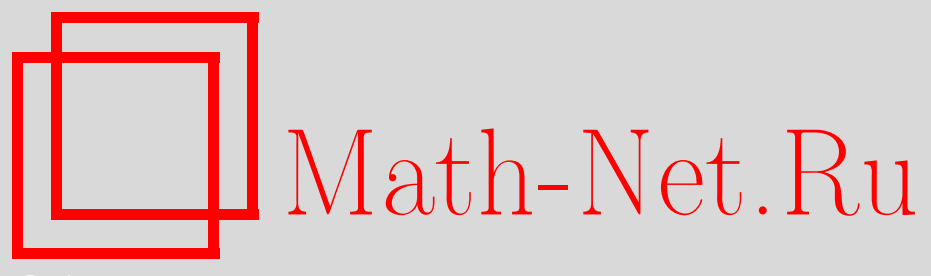

А. Л. Константинов, Инвариантные упорядочения на однородных пространствах с неприводимой группой изотропии, УМН, 2008, том 63, выпуск 2, 171-172

DOI: https://doi.org/10.4213/rm9067

Использование Общероссийского математического портала Math-Net.Ru подразумевает, что вы прочитали и согласны с пользовательским соглашением http: //www. mathnet.ru/rus/agreement

Параметры загрузки:

IP: 18.234 .156 .22

26 апреля 2023 г., 13:29:00

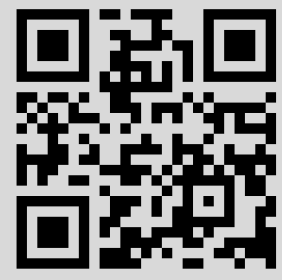




\section{Инвариантные упорядочения на однородных пространствах с неприводимой группой изотропии}

\section{А. Л. Константинов}

1. Введение. Пусть $G$ - простая вещественная группа Ли, $H \subset G$ - подгруппа Ли, которая действует в касательном пространстве однородного пространства $G / H$ неприводимо. Тогда пространство $G / H$ называется однородным пространством с неприводимой группой изотропии. Для краткости мы будем называть такие пространства неприводимыми. В настоящей работе дана полная классификация несимметрических неприводимых однородных пространств, допускающих инвариантные упорядочения, для случая неэрмитовой группы $G$.

Инвариантным упорядочением на однородном пространстве $G / H$ группы Ли $G$ называется частичное упорядочение, инвариантное относительно действия группы $G$. Пусть $\varphi: G \rightarrow G / H$ - каноническое отображение. Инвариантное упорядочение на $G / H$ называется непрерывным, если множество $Q=\{x \in G / H \mid x \geqslant e H\}$ замкнуто и полугруппа $P=\varphi^{-1}(Q)$ порождается любой своей окрестностью единицы. Обратно, любая замкнутая полугруппа $P \subset G$, группа обратимых элементов которой совпадает с $H$, задает инвариантное упорядочение на $G / H$. Далее все инвариантные упорядочения будут считаться непрерывными.

Через $\mathfrak{g}$ и $\mathfrak{h}$ обозначим касательные алгебры групп $G$ и $H$ соответственно. Каждому инвариантному упорядочению на $G / H$ соответствует $\operatorname{Ad} H$-инвариантный замкнутый строго выпуклый конус $C$ в пространстве $\mathfrak{g} / \mathfrak{h}$. Этот конус состоит из всех векторов, касательных к кривым, лежащим в $Q$. Упорядочение однозначно восстанавливается по $C$ [1].

Инвариантные упорядочения на симметрических однородных пространствах простых групп Ли классифицировал Г. И. Ольшанский [1]. Случай однородных пространств эрмитовых групп Ли был рассмотрен в [2].

О. В. Мантуров в работе [3] классифицировал все неприводимые однородные пространства $G / H$ для случая компактной простой группы Ли $G$. Пользуясь теорией Ф. И. Карпелевича [4] о вложении вещественных форм, результаты Мантурова можно обобщить на однородные пространства $G / H$ ( $H$ - редуктивная подгруппа Ли) произвольных простых вещественных групп Ли $G$.

В настоящей работе получен следующий результат.

Теорема. Несимметрические неприводимые однородные пространства неэрмитовых простых вещественных групп Ли, допускающие инвариантные упорядочения, - это в точности однородные пространства, локально изоморфные следующим: $\mathrm{SL}_{n(n+1) / 2}(\mathbb{R}) / \mathrm{PSL}_{n}(\mathbb{R}), \quad \mathrm{SL}_{n^{2}}(\mathbb{R}) / \mathrm{PSL}_{n}(\mathbb{C}), \quad \mathrm{SL}_{n(2 n-1)}(\mathbb{R}) / \mathrm{PSL}_{n}(\mathbb{H}), \quad \mathrm{SL}_{27}(\mathbb{R}) / \mathrm{EIV}$, $\mathrm{EV} / \mathrm{PSL}_{3}(\mathbb{R})$.

Так как группа $G$ неэрмитова, центр ее односвязной накрывающей конечен. Поэтому достаточно доказать существование инвариантного упорядочения для одного пространства из каждого класса локально изоморфных.

2. Краткое доказательство теоремы. Пусть $G$ - неэрмитова простая линейная группа Ли, а $H$ - такая ее полупростая подгруппа Ли, что однородное пространство $G / H$ неприводимо. Их максимальные компактные подгруппы обозначим через $K$ и $L$ соответственно, причем будем считать, что $L \subset K$. Касательные алгебры групп Ли будем обозначать соответствующими маленькими готическими буквами.

Известно [5], что в пространстве $V$ неприводимого представления связной полупростой группы Ли $H$ существует $H$-инвариантный замкнутый выпуклый конус тогда и только тогда, когда существует ненулевой $L$-инвариантный вектор. Если такой вектор существует, то он единствен с точностью до пропорциональности. Таким 
образом, для существования инвариантного упорядочения на $G / H$ необходимо существование ненулевого $\mathrm{Ad} L$-инвариантного вектора $v_{L}$ в пространстве $\mathfrak{g} / \mathfrak{h}$, которое отождествляется с $H$-инвариантным дополнением к $\mathfrak{h}$ в $\mathfrak{g}$. Также для существования инвариантного упорядочения на $G / H$ необходимо, чтобы все собственные значения оператора $\operatorname{ad} v_{L}$ были вещественны.

В таблице приведены все, с точностью до локального изоморфизма, однородные пространства $G / H$ описанного выше типа, для которых в $\mathfrak{g} / \mathfrak{h}$ существует $\mathrm{Ad} H$-инвариантный конус. Во втором столбце указан вес $\Lambda_{h}$ ограничения минимального представления алгебры $\mathfrak{g}_{\mathbb{C}}$ на подалгебру $\mathfrak{h}_{\mathbb{C}}\left(\pi_{i}-i\right.$-й фундаментальный вес, $R(\Lambda)$, $S(\Lambda)$ - неприводимые представления со старшим весом $\Lambda$ ), в третьем столбце указан старший вес $\Lambda_{\text {ad }}$ представления алгебры $\mathfrak{h}$ в $\mathfrak{g} / \mathfrak{h}$, в последнем столбце поставлен знак Re, если вектор $v_{L}$ веществен, и знак $\mathrm{Im}$, если $v_{L}$ чисто мнимый.

\begin{tabular}{|c|c|c|c|}
\hline$G / H$ & $\Lambda_{h}$ & $\Lambda_{\mathrm{ad}}$ & $v_{L}$ \\
\hline $\mathrm{SL}_{\frac{n(n+1)}{2}}(\mathbb{R}) / \mathrm{PSL}_{n}(\mathbb{R})$ & $R\left(2 \pi_{1}\right)$ & $R\left(2 \pi_{1}+2 \pi_{n-1}\right)$ & $\mathrm{Re}$ \\
\hline $\mathrm{SL}_{n^{2}}(\mathbb{R}) / \mathrm{PSL}_{n}(\mathbb{C})$ & $R\left(\pi_{1}\right) \otimes S\left(\pi_{n-1}\right)$ & $R\left(\pi_{1}+\pi_{n-1}\right) \otimes S\left(\pi_{1}+\pi_{n-1}\right)$ & $\mathrm{Re}$ \\
\hline $\mathrm{SL}_{n(2 n-1)}(\mathbb{R}) / \mathrm{PSL}_{n}(\mathbb{H})$ & $R\left(\pi_{2}\right)$ & $R\left(\pi_{2}+\pi_{n-2}\right)$ & $\mathrm{Re}$ \\
\hline $\mathrm{SL}_{27}(\mathbb{R}) / \mathrm{EIV}^{2}$ & $R\left(\pi_{1}\right)$ & $R\left(\pi_{1}+\pi_{5}\right)$ & $\mathrm{Re}$ \\
\hline $\mathrm{SO}_{38,32}^{0} / \mathrm{PSU}_{4,4}$ & $R\left(\pi_{4}\right)$ & $R\left(\pi_{3}+\pi_{5}\right)$ & $\mathrm{Im}$ \\
\hline $\mathrm{SO}_{12,8}^{0} / \mathrm{PSU}_{2,2}$ & $R\left(2 \pi_{2}\right)$ & $R\left(\pi_{1}+2 \pi_{2}+\pi_{3}\right)$ & $\mathrm{Im}$ \\
\hline $\mathrm{SO}_{2 n^{2}-n+2,4 n-2}^{0} / \mathrm{PSO}_{2 n-1,2}^{0}$ & $R\left(2 \pi_{1}\right)$ & $R\left(2 \pi_{1}+\pi_{2}\right)$ & $\mathrm{Im}$ \\
\hline $\mathrm{SO}_{22,20}^{0} / \mathrm{PSp}_{8}(\mathbb{R})$ & $R\left(\pi_{4}\right)$ & $R\left(2 \pi_{3}\right)$ & $\mathrm{Im}$ \\
\hline $\mathrm{SO}_{2 n^{2}+n+2,4 n}^{0} / \mathrm{PSO}_{2 n, 2}^{0}$ & $R\left(2 \pi_{1}\right)$ & $R\left(2 \pi_{1}+\pi_{2}\right)$ & $\mathrm{Im}$ \\
\hline $\mathrm{PSO}_{72,56}^{0} / \mathrm{PSO}_{16}^{*}$ & $R\left(\pi_{8}\right)$ & $R\left(\pi_{6}\right)$ & $\mathrm{Im}$ \\
\hline $\mathrm{GI}_{2} \mathrm{PSL}_{2}(\mathbb{R})$ & $R\left(6 \pi_{1}\right)$ & $R\left(10 \pi_{1}\right)$ & $\mathrm{Re}$ \\
\hline $\mathrm{EV} / \mathrm{PSL}_{3}(\mathbb{R})$ & $R\left(6 \pi_{1}\right)+R\left(6 \pi_{2}\right)$ & $R\left(4 \pi_{1}+4 \pi_{2}\right)$ & $\mathrm{Im}$ \\
\hline $\mathrm{EVI} / \mathrm{PSU}_{2,1}$ & $R\left(6 \pi_{1}\right)+R\left(6 \pi_{2}\right)$ & $R\left(4 \pi_{1}+4 \pi_{2}\right)$ & \\
\hline
\end{tabular}

Остается доказать, что случаях, когда вектор $v_{L}$ веществен, инвариантное упорядочение существует. Во всех пяти случаях ограничение представления группы $G$ на подгруппу $H$ имеет инвариантный выпуклый конус.

Рассмотрим любой из этих конусов. Обозначим его через $D$, а лежащий в нем $L$-инвариантный вектор обозначим через $v_{D}$. Рассмотрим полугруппу $P=\{g \in G \mid$ $g D \subset D\}$ сжатий конуса $D$. Подгруппа обратимых элементов полугруппы $P$ совпадает с группой $H$, а сама полугруппа $P$ имеет непустую внутренность. Касательный конус к ней обозначим через $C$. Полугруппа $\overline{\langle\exp C\rangle}$ задает некоторое нетривиальное инвариантное упорядочение на $G / H$.

\section{Список литературы}

[1] Г. И. Ольшанский, Докл. АН ССCР, 265:3 (1982), 537-541; англ. пер.: G. I. Ol'shanskii, Soviet Math. Dokl., 26:1 (1982), 97-101. [2] А. Л. Константинов, Вестн. Моск. ун-та. Сер. 1. Матем., мех., 2006, №6, 15-18. [3] О. В. Мантуров, Тр. сем. по векторному и тензорному анализу, 13, Изд-во МГУ, 1966, 68-145. [4] Ф. И. Карпелевич, Тр. ММО, 4 (1955), 3-112. [5] Э. Б. Винберг, Функи. анализ и его прил., 14:1 (1980), 1-13; англ. пер.: Ё. В. Vinberg, Funct. Anal. Appl., 14:1 (1980), 1-10.

А. Л. Константинов (А. L. Konstantinov)

Московский государственный университет им. М. В. Ломоносова

E-mail: lelik_msu@rambler.ru
Представлено Э. Б. Винбергом Принято редколлегией 07.12 .2007 